\title{
Kähler structure in the commutative limit of matrix geometry
}

\author{
Goro Ishiki, ${ }^{a, b}$ Takaki Matsumoto $^{b}$ and Hisayoshi Muraki ${ }^{b}$ \\ ${ }^{a}$ Center for Integrated Research in Fundamental Science and Engineering (CiRfSE), \\ University of Tsukuba, \\ Tsukuba, Ibaraki 305-8571, Japan \\ ${ }^{b}$ Graduate School of Pure and Applied Sciences, University of Tsukuba, \\ Tsukuba, Ibaraki 305-8571, Japan \\ E-mail: ishiki@het.ph.tsukuba.ac.jp, matsumoto@het.ph.tsukuba.ac.jp, \\ hmuraki@het.ph.tsukuba.ac.jp
}

ABSTRACT: We consider the commutative limit of matrix geometry described by a large$N$ sequence of some Hermitian matrices. Under some assumptions, we show that the commutative geometry possesses a Kähler structure. We find an explicit relation between the Kähler structure and the matrix configurations which define the matrix geometry. We also discuss a relation between the matrix configurations and those obtained from the geometric quantization.

KEYwords: M(atrix) Theories, Non-Commutative Geometry

ArXiv ePrint: 1603.09146 


\section{Contents}

1 Introduction 1

2 Classical limit of matrix geometry 3

2.1 Classical space 3

2.2 Tangent space 5

2.3 Local coordinates 6

$\begin{array}{llr}3 & \text { Kähler structure } & 7\end{array}$

$\begin{array}{ll}3.1 \text { Poisson structure } & 7\end{array}$

$\begin{array}{lll}3.2 & \text { Symplectic structure } & 7\end{array}$

3.3 Complex structure 8

$\begin{array}{lll}3.4 & \text { Riemann structure } & 10\end{array}$

4 An example: fuzzy sphere $\quad 10$

5 Relation to the geometric quantization $\quad 11$

$\begin{array}{lll}6 & \text { Summary and discussions } & 13\end{array}$

$\begin{array}{ll}\text { A Projectivity of } P^{\mu}{ }_{\nu} & 14\end{array}$

B Properties of $W^{\mu \nu} \quad 16$

$\begin{array}{lll}\text { B.1 Proof of (3.2) } & 16\end{array}$

$\begin{array}{ll}\text { B.2 Proof of Jacobi identity } & 17\end{array}$

$\begin{array}{ll}\text { C Construction of compatible structures } & 18\end{array}$

$\begin{array}{ll}\text { D Explicit forms of } J \text { and } \tilde{g} & 19\end{array}$

$\begin{array}{ll}\text { E Geometric quantization on Kähler manifold } & 19\end{array}$

\section{Introduction}

The matrix geometry is a sort of quantized geometry. It appears naturally in describing D-branes or membranes in string theories and is expected to play important roles in formulating the superstring theory and M-theory [1-3]. The matrix geometry is also closely related to interesting fields in mathematics such as the noncommutative geometry and the deformation/geometric quantization.

In this paper, we focus on the matrix-geometric description of a manifold embedded in the flat $D$-dimensional space and understand a relation to the usual differential geometric 
description of the manifold. The matrix geometry for an embedded space in $R^{D}$ can be defined in terms of $D$ Hermitian matrices $X^{\mu}$. They can be considered as the quantized version of an embedding function $y: \mathcal{M} \rightarrow R^{D}$. In fact, in some well-known examples such as the fuzzy sphere [4], these matrices can be constructed explicitly from the given embedding function through the geometric quantization. For example, see $[5,6]$ and references therein for this topic.

For matrices with a fixed size, one can extract geometric information by using the methods proposed in [7] (see also [8]). Here, the corresponding geometry is defined as loci of zero eigenmodes of the matrix version of the Dirac operator, $\gamma_{\mu}\left(X^{\mu}-y^{\mu}\right)$ (or the Laplacian $\left(X^{\mu}-y^{\mu}\right)^{2}$ can also be used as considered in [8]), where $y \in R^{D}$ are parameters. This definition is natural from the view point of the string theory. If $X^{\mu}$ describe a certain shape of a D-brane, the Dirac operator appears in the low-energy effective action of open strings connecting the D-brane with another probe brane at the position $y$. The zero eigenmodes are just massless modes of open strings. Thus at that position, the probe brane feels the presence of the D-brane. So the set of zero eigenstates can naturally be identified with the shape of the D-brane.

Here we also remark that a similar prescription can also be found in a different context $[9,10]$. Here the Dirac operator corresponds to a tachyon configuration in a non-BPS brane system and the set of zero eigenmodes corresponds to the shape of the resultant stable D-brane after the tachyon condenses.

In this paper, in contrast to the above mentioned work, we consider a large- $N$ family of $X^{\mu}$, not just the matrices with a fixed size. Namely, our starting point is that $\left\{X_{\mu}^{(N)} \mid N \in I\right\}$ is given, where $X_{\mu}^{(N)}(\mu=1, \cdots, D)$ are $N \times N$ Hermitian matrices and $I$ is a fixed index set made of a strictly increasing infinite sequence of natural numbers. Then assuming that the commutative limit is a smooth manifold, we try to extract information of the commutative manifold from the given matrix configurations. In this setup, a description of the commutative space, its tangent space and Poisson structure was given in [11] based on the notion of the coherent states. We investigate this method further in this paper.

The motivation to consider this setup partially lies in the matrix regularization, which plays an important role in formulating M-theory. The matrix regularization is a method of approximating a functional ring $C(\mathcal{M})$ on a symplectic manifold $\mathcal{M}$ using the matrix algebra $M_{N}(C)$. The regularization is characterized by a sequence $\left\{T_{N}\right\}$ of linear maps $T_{N}: C(\mathcal{M}) \rightarrow M_{N}(C)$ such that in the large- $N$ limit the algebraic structure is preserved and the Poisson bracket of two functions is mapped to the commutator of matrices, namely, $\left\|T_{N}(f) T_{N}(g)-T_{N}(f g)\right\| \rightarrow 0$ and $\left\|T_{N}(\{f, g\})-i N\left[T_{N}(f), T_{N}(g)\right]\right\| \rightarrow 0$. See [12] for more detailed treatment. If we regard $X^{\mu}$ as the image of the embedding function, our problem of finding the geometry from given matrices is just the inverse problem of the construction of matrix regularization. Understanding the inverse problem should shed some light on the construction problem of the matrix regularization.

In this paper, for the given sequence of matrices, we consider how to recover the differential geometry of the manifold in the commutative limit. We focus on matrices 
which satisfy

$$
\left[X^{\mu}, X^{\nu}\right]=\frac{i}{C_{N}} W^{\mu \nu}(X)+\cdots,
$$

where, $W^{\mu \nu}(X)$ is a polynomial of $X^{\mu}$ such that its definition (degree and coefficients) does not depend on $N . C_{N}$ is a real $N$-dependent constant which goes to infinity as $N \rightarrow \infty$ and $\cdots$ in (1.1) stands for higher order terms in $1 / C_{N}$. The large- $N$ limit thus corresponds to the commutative limit of the matrix geometry. We also call this limit classical limit in this paper since this corresponds to the limit of $\hbar \rightarrow 0$ in quantum mechanics, where $X^{1}=\hat{p}$ and $X^{2}=\hat{q}$.

We show that if the matrices $X^{\mu}$ satisfy (1.1) the associated classical space possesses not only a Poisson structure but also a Kähler structure. ${ }^{1}$ We also argue that when $C_{N}$ satisfies a certain condition, the matrices which satisfy (1.1) can be semi-classically equivalent to those obtained from the geometric quantization scheme.

The condition (1.1) strongly restricts possible form of the matrices. However, this condition is satisfied by most known symmetric examples of matrix geometry such as the fuzzy $C P^{n}$ [13] and fuzzy torus [14]. See also [15] for a less symmetric example which satisfies (1.1). ${ }^{2}$ Moreover, even if one adds a perturbation given by $N$-independent polynomials in $X^{\mu}$ to configurations satisfying (1.1), still the condition is kept satisfied up to any order of the perturbation. Thus, this setup is also relevant for theories expanded around some fixed background configuration.

This paper is organized as follows. In section 2, we review [11] and see the description of the classical space and its tangent space in terms of given matrix elements. In section 3 , we show that when (1.1) is satisfied, the classical space possesses a Kähler structure. We also relate the geometric structure to the matrix configurations. In section 4 , we consider the fuzzy sphere as an example and show explicit forms of the geometric objects defined in the previous section. In section 5, we discuss a relation to the geometric quantization. Section 6 is devoted to a summary and discussions.

\section{Classical limit of matrix geometry}

In this section, we review [11] and recall the description of the classical space and its tangent space in terms of the given matrix elements.

\subsection{Classical space}

As we described in the previous section, we assume that configurations of the coordinate matrices, $\left\{X_{\mu}^{(N)} \mid N \in I\right\}$, is given. In the following, we will omit the superscript $(N)$.

We adopt the definition of the classical space proposed in [11]. We first define an $N \times N$ Hermitian matrix $H$, which we call the Hamiltonian, as

$$
H(y)=\frac{1}{2}\left(X^{\mu}-y^{\mu} \mathbf{1}\right)^{2} .
$$

\footnotetext{
${ }^{1}$ To conclude this, we also assume one more condition that the ground state of the defining Hamiltonian of the classical geometry is non-degenerate. This is just a technical assumption and will be explained in the following sections.

${ }^{2}$ On the other hand, (1.1) is not satisfied for the fuzzy (real) four sphere [16] for example.
} 
where $y^{\mu}(\mu=1,2, \ldots, D)$ are real parameters. Then, we define the classical space as the loci of zeros of the ground state energy $E_{0}(y)$ of $H(y)$ in the classical limit $N \rightarrow \infty$. More specifically, we write

$$
\mathcal{M}=\left\{y \in R^{D} \mid f(y)=0\right\},
$$

where the function $f(y)$ is the large- $N$ limit of the ground state energy,

$$
f(y)=\lim _{N \rightarrow \infty} E_{0}(y) .
$$

One can understand this definition as follows. The ground state energy of the Hamiltonian can be written as

$$
E_{0}(y)=\frac{1}{2}\left(\Delta X^{\mu}\right)^{2}+\frac{1}{2}\left(\left\langle X^{\mu}\right\rangle-y^{\mu}\right)^{2},
$$

where $\Delta X^{\mu}$ and $\left\langle X^{\mu}\right\rangle$ are the standard deviation and expectation value of $X^{\mu}$ with respect to the ground state, respectively. When $E_{0}(y)$ is vanishing, both $\Delta X^{\mu}$ and $\left\langle X^{\mu}\right\rangle-y^{\mu}$ are vanishing. Thus, it follows that if the ground state energy vanishes, there exists a wave packet which shrinks to that point. It is easy to show that the converse is also true. Therefore, the space $\mathcal{M}$ defined by (2.2) can be regarded as a set of points in $R^{D}$ such that there exist shrinking wave functions, which can naturally be identified with the classical points. ${ }^{3}$

There is an ambiguity in the definition of the Hamiltonian. For example, if one takes the square of $H$ as the new Hamiltonian, the above arguments about the shrinking wave packets will still hold. Since the classical geometries described by those different Hamiltonians are the same, one may use either of those definitions. However, the definition (2.1) has two advantages. One is just the simplicity. Our Hamiltonian is the simplest choice among those which are compatible with the translational and rotational symmetries of $R^{D}$. This will make it easier to compute the geometry and the geometric objects discussed in the next section from the given matrices. The other advantage is in the context of the string theory. We can find some physical origins of (2.1) in some systems with D-branes. For example, we can regard (2.1) as the Laplacian in the low energy effective action of open strings in a D-brane system with a probe D0-brane [7] (see also [20]). The classical space then corresponds to the shape of a D-brane (or a membrane) in the target space. Thus, the definition (2.1) allows a clear physical interpretation.

For a general (random) sequence of matrices, the classical space (2.2) is the most likely to be a non-manifold or an empty set. A necessary condition to have a non-empty set is that the matrices $X^{\mu}$ become commuting with each other in the large- $N$ limit, since the ground state energy is bounded from below by $\left|\left\langle\left[X^{\mu}, X^{\nu}\right]\right\rangle\right| / 2$, where $\mu$ and $\nu$ are arbitrary. This can be shown in the same way as the uncertainty inequality in the quantum mechanics. Since we assume (1.1) in this paper, this necessary condition is satisfied.

The relation (1.1) also allows us to relate the definition of the classical space with that in [7]. In [7], surfaces embedded in the three dimensional flat space are considered. ${ }^{4}$ Here,

\footnotetext{
${ }^{3}$ Those wave functions correspond to the coherent states in quantum mechanics. See [17-19] for applications of coherent states in describing matrix geometries.

${ }^{4}$ See also [21] for an application to describing various configurations of membranes and see [22] for a higher dimensional extension.
} 
the classical space is defined for any fixed $N$ as the loci of zero eigenvectors of the Dirac operator $D(y)=\sigma_{i}\left(X^{i}-y^{i}\right)$, where $\sigma_{i}$ are the Pauli matrices. Note that the square of the Dirac operator gives our Hamiltonian plus terms proportional to the commutators of $X^{\mu}$. If the condition (1.1) is satisfied, the commutators become negligible in the large- $N$ limit. Hence, in the large $N$ limit, the classical space defined by the Hamiltonian is equivalent to the space defined by the Dirac operator.

This fact allows us to use the method proposed in [7] to check whether the configuration defines a smooth geometry or not, at least for some particular cases. In this method, one considers an index defined from the matrix Dirac operator. If the index is non-vanishing, it is assured that the classical space is a smooth surface embedded in the flat space.

Though this can be applied in our setup, we will not consider this problem further in the following. Instead, we assume that the classical space is a smooth manifold such as those having non-vanishing values of the index. More precisely speaking, we assume that $\mathcal{M}$ is a non-empty connected subset of $R^{D}$ and there exists a neighbourhood of $\mathcal{M}$ on which the function $f(y)$ is smooth. The latter condition is required for $\mathcal{M}$ to be smooth and to have a well defined tangent space, as we will see in the next subsection.

\subsection{Tangent space}

In order to develop a theory of differential geometry on $\mathcal{M}$, here we define the tangent space of $\mathcal{M}$ in terms of the given matrices. At each point $y \in \mathcal{M}$, let us consider an arbitrary $D$ dimensional vector $B^{\mu}(y)$. There should exist a projection operator, which projects $B^{\mu}(y)$ onto its tangent components along $\mathcal{M}$. The projection operator is given by [11]

$$
P^{\mu}{ }_{\nu}(y)=\delta_{\nu}^{\mu}-\partial^{\mu} \partial_{\nu} f(y) \text {. }
$$

Here and thereafter, we raise and lower the $D$-dimensional indices by the Kronecker delta (the flat metric on the target space), so that $\partial^{\mu}=\partial_{\mu}$. See appendix A for a proof of the projectivity of $P^{\mu}{ }_{\nu}$. In terms of the projection operator, the tangent vectors are defined by the relation,

$$
P_{\nu}^{\mu}(y) B^{\nu}(y)=B^{\mu}(y)
$$

for $y \in \mathcal{M}$.

The projection operator is positive definite on the tangent vectors by definition and hence it can be regarded as a metric on $\mathcal{M}$. This metric corresponds to the induced metric (the closed string metric [23]) associated with the original embedding $\mathcal{M} \rightarrow R^{D}$.

The projection $P^{\mu}{ }_{\nu}$ can be computed from the given matrices by using the perturbation theory in quantum mechanics, as follows. For a sufficiently small shift of the variables, $y^{\mu} \rightarrow y^{\mu}+\epsilon^{\mu}$, we have $H(y+\epsilon)=H(y)+\epsilon \cdot(y-X)+\frac{1}{2} \epsilon^{2}$. The deviation of the ground state energy can be computed by treating the terms with $\epsilon^{\mu}$ as perturbation. The large$N$ limits of the perturbative corrections for the ground state energy correspond to the derivatives of $f(y)$, which give $P^{\mu}{ }_{\nu}$. More specifically, by introducing the eigenstates of $H(y)$ as

$$
H(y)|n, y\rangle=E_{n}(y)|n, y\rangle,
$$


the projection operator can be written as

$$
P_{\nu}^{\mu}(y)=2 \lim _{N \rightarrow \infty} \sum_{n \neq 0} \operatorname{Re} \frac{\left\langle 0, y\left|X^{\mu}\right| n, y\right\rangle\left\langle n, y\left|X_{\nu}\right| 0, y\right\rangle}{E_{n}(y)-E_{0}(y)} .
$$

This expression provides a direct relation between the matrix elements and the geometric object $\left\{P_{\nu}^{\mu}\right\}$. Here, in order to write down the formula (2.8), we have assumed that the ground state is non-degenerate on $\mathcal{M}$. The case with degenerate ground states will be briefly discussed in the last section.

For later convenience, we write some useful relations below. For any normalized vector $|\psi\rangle$, we have

$$
\lim _{N \rightarrow \infty}\left\langle\psi\left|y^{\mu}-X^{\mu}\right| 0, y\right\rangle=0 . \quad(y \in \mathcal{M})
$$

This follows from the Cauchy-Schwarz inequality and the fact that $\lim _{N \rightarrow \infty} E_{0}(y)=0$ for $y \in \mathcal{M}$. This relation can be generalized to

$$
\lim _{N \rightarrow \infty}\langle\psi|h(y)-h(X)| 0, y\rangle=0, \quad(y \in \mathcal{M})
$$

where $h(y)$ is any polynomial with the coefficients and degree $N$-independent. Here, we have assumed that $X_{\mu}$ are norm-bounded in the large- $N$ limit.

\subsection{Local coordinates}

We can introduce at least locally some parameters $\sigma^{a}$ which solve $f(y(\sigma))=0$. The vectors of the form

$$
e_{a}^{\mu}(\sigma)=\frac{\partial y^{\mu}(\sigma)}{\partial \sigma^{a}}
$$

are tangent vectors satisfying (2.6). Since we have assumed that $\mathcal{M}$ is a manifold, there exists a parametrization such that $\sigma^{a}$ are local coordinates on $\mathcal{M}$, where $a$ runs from 1 to the dimension of $\mathcal{M}$. In this case, the vectors (2.11) form a basis of the tangent vectors at $y(\sigma) \in \mathcal{M}$ and the symmetric tensor

$$
g_{a b}(\sigma)=\left(e_{a}(\sigma), e_{b}(\sigma)\right)=\frac{\partial y^{\mu}(\sigma)}{\partial \sigma^{a}} \frac{\partial y_{\mu}(\sigma)}{\partial \sigma^{b}}
$$

is non-degenerate, where $($,$) is the natural inner product of the D$-dimensional vectors in $R^{D}$.

Any tangent vector $B^{\mu}$ can then be expanded using (2.11) as

$$
B^{\mu}=B^{a} e_{a}^{\mu} .
$$

The coefficients $B^{a}$ are uniquely determined from $B^{\mu}$, once the basis is fixed. Similarly, for a given differential form on $R^{D}$, we can also consider a pullback onto $\mathcal{M}$. For example, a one-form $B=B_{\mu} d y^{\mu}$ gives a one-form $B_{a} d \sigma^{a}$ on $\mathcal{M}$, where $B_{a}=B_{\mu} e_{a}^{\mu}$. Thus by using the local coordinates, we can always move to the usual convention that the indices of differential forms and tangent vectors run from 1 to the dimension of the manifold under consideration. For example, (2.12) is nothing but the induced metric $\delta_{\mu \rho} P^{\rho}{ }_{\nu}$ written in terms of the local coordinates.

In this way, switching the conventions of indices is always possible, once local coordinates are introduced. However, we will mainly work with the indices $\mu, \nu$ in this paper to avoid complexity of introducing the local coordinates. 


\section{Kähler structure}

In this section, we show that when the condition (1.1) is satisfied, the classical space $\mathcal{M}$ possesses a Kähler structure, which is a compatible triple of Riemann, symplectic and complex structures.

\subsection{Poisson structure}

Before we consider the Kähler structure, we introduce a Poisson structure, which becomes very important in this section. Let us consider a $D \times D$ real antisymmetric matrix defined $\mathrm{as}^{5}$

$$
W^{\mu \nu}(y)=-i \lim _{N \rightarrow \infty} C_{N}\left\langle 0, y\left|\left[X^{\mu}, X^{\nu}\right]\right| 0, y\right\rangle .
$$

For $y \in \mathcal{M}$, one can see that (3.1) gives a Poisson tensor on $\mathcal{M}$ [11]. Namely, it is a tangent bivector on $\mathcal{M}$ satisfying

$$
P^{\mu}{ }_{\nu}(y) W^{\nu \rho}(y)=W^{\mu \rho}(y), \quad(y \in \mathcal{M})
$$

and the Poisson bracket defined by

$$
\{f, g\}=W^{\mu \nu}\left(\partial_{\mu} f\right)\left(\partial_{\nu} g\right)
$$

satisfies the Jacobi identity. See appendix B for a proof of the above statements.

\section{$3.2 \quad$ Symplectic structure}

Since our Hamiltonian depends on the parameters $y^{\mu}$, we can introduce the notion of the Berry phase and the Berry curvature. Here, we show that under our assumptions, the Berry curvature of the Hamiltonian (2.1) gives a symplectic form on $\mathcal{M}$.

Under an infinitesimal shift of the parameters, $y^{\mu} \rightarrow y^{\mu}+\epsilon^{\mu}$, the ground state of the Hamiltonian (2.1) varies by $|0, y\rangle \rightarrow|0, y\rangle+\epsilon^{\mu} \partial_{\mu}|0, y\rangle$, where $\partial_{\mu}$ here means a linear map on the Hilbert space defined by (B.9), which is just the first order formula of the perturbation theory. $A_{\mu}(y) \in R$ in (B.9) is a real c-number and called the Berry connection. By a phase rotation of the ground state, $A_{\mu}(y)$ transforms as a gauge field. Though at least locally one can eliminate $A_{\mu}(y)$ by a suitable gauge transformation, the curvature is gauge invariant and is nonvanishing in general.

It is appropriate to define the covariant derivative on the Hilbert space as

$$
D_{\mu}=\partial_{\mu}-i A_{\mu}
$$

Then the Berry curvature is defined as the field strength of $A_{\mu}(y)$ :

$$
F_{\mu \nu}=i\left[D_{\mu}, D_{\nu}\right]
$$

\footnotetext{
${ }^{5}$ Note that we use the same notation for this matrix and the polynomial in the right-hand side of (1.1). This is because the matrix (3.1) is indeed obtained by replacing $X^{\mu}$ with $y^{\mu}$ in the polynomial in (1.1). See (2.10).
} 
$F_{\mu \nu}$ is a real number and can also be written as

$$
F_{\mu \nu}(y)=2 \sum_{n \neq 0} \operatorname{Im} \frac{\left\langle 0, y\left|X_{\mu}\right| n, y\right\rangle\left\langle n, y\left|X_{\nu}\right| 0, y\right\rangle}{\left(E_{n}(y)-E_{0}(y)\right)^{2}} .
$$

Note that $F_{\mu \nu}$ is defined for each fixed $N$ unlike $P^{\mu}{ }_{\nu}$ and $W^{\mu \nu}$, which are defined only in the large- $N$ limit.

We define a 2 -form on $R^{D}$ by

$$
\omega_{\mu \nu}(y)=-\lim _{N \rightarrow \infty} \frac{F_{\mu \nu}(y)}{C_{N}}=-\lim _{N \rightarrow \infty} \frac{2}{C_{N}} \sum_{n \neq 0} \operatorname{Im} \frac{\left\langle 0, y\left|X_{\mu}\right| n, y\right\rangle\left\langle n, y\left|X_{\nu}\right| 0, y\right\rangle}{\left(E_{n}(y)-E_{0}(y)\right)^{2}} .
$$

The pullback of this 2-form gives a symplectic form on $\mathcal{M}$ as we will show below. Firstly, it satisfies

$$
W^{\mu \rho}(y) \omega_{\rho \nu}(y)=P_{\nu}^{\mu}(y) . \quad(y \in \mathcal{M})
$$

Namely, on tangent vectors it gives the inverse of the Poisson tensor. This guarantees that the pullback is non-vanishing everywhere on $\mathcal{M}$. Secondly, it satisfies $d \omega=0$ or equivalently,

$$
\partial_{\mu} \omega_{\nu \rho}(y)+(\text { cyclic permutation })=0 . \quad(y \in \mathcal{M})
$$

Since taking the pullback and exterior derivative commute, this implies that the pullback is closed. The proof of (3.8) is almost the same as that for (3.2) shown in appendix B, while (3.9) follows immediately from the definition of the Berry curvature (3.5). From these properties we find that the pullback of $\omega$ gives a symplectic form on $\mathcal{M}$.

The Berry connection has a physical interpretation in string theories. By investigating a simple example like a fuzzy sphere plus fluctuations, we can find that the Berry connection corresponds to the gauge fields on D-branes. Let us consider Myer's effect [24] and regard the fuzzy sphere as the blowing up effect from D0-branes to spherical D2-branes. The fluctuations on D0-branes can be decomposed to the tangent and normal components on the sphere. ${ }^{6}$ In our setup, through a direct computation, we can see that the tangent components give a fluctuation of the Berry connection $A_{\mu}$. On the other hand, from the action of D0-branes, we can see that the tangent components become the gauge fields on the D2-branes $[26,27]$. This suggests that $A_{\mu}$ can be identified with the gauge fields on the emergent D-branes.

From (3.7) and (3.8), we see that the gauge fields have a field strength $F_{\mu \nu}$ with magnetic flux of order $C_{N}$, which goes to infinity in the large- $N$ limit. Since the presence of magnetic flux is equivalent to a nontrivial B-field background in the Dirac-Born-Infeld action, the large- $N$ commutative limit corresponds to a limit of a very strong B-field background in this context.

\subsection{Complex structure}

For a given symplectic structure and an arbitrary metric, one can construct an almost complex structure, which is compatible with the symplectic structure, as shown in appendix C.

\footnotetext{
${ }^{6}$ See $[25]$ for example.
} 
Since now we have the symplectic structure (3.7) and the induced metric (2.12) on $\mathcal{M}$, we can also obtain a compatible almost complex structure on $\mathcal{M}$. It is given by

$$
J(y)=\frac{1}{\sqrt{W(y) W^{T}(y)}} W(y) .
$$

Here, $W$ denotes a $D \times D$ antisymmetric real matrix with the matrix elements given by $W^{\mu}{ }_{\nu}=\delta_{\nu \rho} W^{\mu \rho}$ and the product in (3.10) is just the matrix product. The inverse power of $W, \frac{1}{\sqrt{W W^{T}}}$, is defined as follows. In general, from (3.2), $W$ has vanishing eigenvalues on the space of normal vectors on $\mathcal{M}$ (the vectors which vanish under a multiplication of $P_{\nu}^{\mu}$.). The inverse of $\frac{1}{\sqrt{W W^{T}}}$ is taken only in the space of the tangent vectors on which $W$ is non-degenerate because of (3.8), leaving the zero eigenvalues as they are.

More specifically, for each $y \in \mathcal{M}$, one can take a suitable basis such that $W$ becomes the canonical form which consists of some $2 \times 2$ diagonal blocks as well as some zero eigenvalues. Denoting the transition matrix to such basis by $V$, we can write

$$
\begin{gathered}
\left\{P_{\nu}^{\mu}\right\}=V\left(\begin{array}{cc}
0 & \\
& 1
\end{array}\right) V^{-1}, \\
\left\{W^{\mu}{ }_{\nu}\right\}=V\left(\begin{array}{cc}
0 & \\
& \tilde{W}
\end{array}\right) V^{-1},
\end{gathered}
$$

where the up-left and the bottom-right blocks correspond to the spaces of the normal and the tangent vectors with dimensions $D-\operatorname{dim} \mathcal{M}$ and $\operatorname{dim} \mathcal{M}$, respectively. Because of (3.8), $\tilde{W}$ is a nondegenerate real antisymmetric matrix, which consists of $2 \times 2$ diagonal blocks. Then, $\frac{1}{\sqrt{W W^{T}}}$ is defined by

$$
\frac{1}{\sqrt{W W^{T}}}=V\left(\begin{array}{l}
0 \\
\frac{1}{\sqrt{\tilde{W} \tilde{W}^{T}}}
\end{array}\right) V^{-1} .
$$

One can easily check that (3.10) satisfies

$$
P(y) J(y)=J(y) P(y)=J(y), \quad J^{2}(y)=-P(y) . \quad(y \in \mathcal{M})
$$

The first equation guarantees that $J$ is a linear map from tangent vectors to themselves and the second equation says that $J^{2}$ is equal to the minus of the identity on the tangent space. Thus, $J$ defines an almost complex structure on $\mathcal{M}$.

Furthermore, we can show that $J$ is integrable, so it gives a complex structure on $\mathcal{M}$. To show this, let us consider differentiating the first equation in (3.11). We obtain

$$
\partial_{\rho} P^{\mu}{ }_{\nu}+\left[\Gamma_{\rho}, P\right]^{\mu}{ }_{\nu}=0
$$

where the derivative shall be restricted to tangential directions on $\mathcal{M}$ and

$$
\Gamma_{\rho}=V \partial_{\rho} V^{-1} .
$$

This expression motivates us to define a covariant derivative as,

$$
\nabla_{\rho} B^{\mu}=\partial_{\rho} B^{\mu}+\left(\Gamma_{\rho}\right)_{\nu}^{\mu} B^{\nu}, \quad \nabla_{\rho} B_{\mu}=\partial_{\rho} B_{\mu}-\left(\Gamma_{\rho}\right)_{\mu}^{\nu} B_{\nu} .
$$


Then (3.14) is written as $\nabla P=0$. Since the tangent space of $\mathcal{M}$ is characterized by $P^{\mu}{ }_{\nu}$ as in (2.6), the equation $\nabla P=0$ guarantees that $\nabla$ is a linear map from tangent vectors to themselves. So it is indeed a covariant derivative on $\mathcal{M}$ preserving inner products with respect to the induced metric. Now, let us consider the covariant derivative of the almost complex structure (3.10). From (3.12) and the second equation in (3.11), it is obvious that

$$
\nabla_{\rho} J_{\nu}^{\mu}=0
$$

Namely, under any parallel transport defined by $\nabla$, the reference of the holomorphicity set by $J_{\nu}^{\mu}$ is preserved. This is nothing but the integrability of $J_{\nu}^{\mu}$.

The complex structure $J$ can also be written in terms of the matrix elements as

$$
J_{\nu}^{\mu}=2 \lim _{N \rightarrow \infty} \sum_{n \neq 0} \operatorname{Im} \frac{\left\langle 0, y\left|X^{\mu}\right| n, y\right\rangle\left\langle n, y\left|X_{\nu}\right| 0, y\right\rangle}{E_{n}(y)-E_{0}(y)} .
$$

This is proved in appendix D.

\subsection{Riemann structure}

For a given compatible pair of a symplectic form and an almost complex structure $(\omega, J)$, one can find a compatible Riemannian metric $\tilde{g}$. See appendix C. In our case, the metric is given by

$$
\tilde{g}=\frac{1}{\sqrt{W W^{T}}} .
$$

Since $J$ is integrable, the compatible triple $(\omega, J, \tilde{g})$ gives a Kähler structure of $\mathcal{M}$.

The compatible metric $\tilde{g}$ can also be written in terms of the given matrix elements as

$$
\tilde{g}_{\mu \nu}=\lim _{N \rightarrow \infty} \frac{2}{C_{N}} \sum_{n \neq 0} \operatorname{Re} \frac{\left\langle 0, y\left|X_{\mu}\right| n, y\right\rangle\left\langle n, y\left|X_{\nu}\right| 0, y\right\rangle}{\left(E_{n}(y)-E_{0}(y)\right)^{2}} .
$$

This is shown in appendix D.

The compatible metric can be interpreted as the information metric of the ground states $\{|0, y\rangle \mid y \in \mathcal{M}\}$. In general, if states are labelled by some parameters $\left\{\lambda^{A} \mid A=1,2, \cdots\right\}$ as $|\lambda\rangle$, the information metric (the Bures distance) between those states are defined by $1-\left|\left\langle\lambda \mid \lambda^{\prime}\right\rangle\right|^{2}$. In our case, by putting $|\lambda\rangle=|0, y\rangle$ and $\left|\lambda^{\prime}\right\rangle=|0, y+d y\rangle$, we can see that the information metric takes exactly the same form as (3.20).

The metric (3.19) also has a physical interpretation. As discussed in section 3.2, the symplectic form can be identified with the B-field on D-branes up to an overall constant and the large- $N$ limit corresponds to a very strong B-field background. The square of the compatible metric $\tilde{g}$ then takes the form $\left(\tilde{g}^{2}\right)_{\mu \nu} \propto B_{\mu \rho} \delta^{\rho \sigma} B_{\sigma \nu}$. This is nothing but the open string metric in the strong B-field limit discussed in [23].

\section{An example: fuzzy sphere}

In this section, we consider the fuzzy sphere and give explicit forms of the geometric objects defined in the previous section. 
The fuzzy sphere is defined by

$$
X^{\mu}=\frac{2}{\sqrt{N^{2}-1}} L^{\mu}
$$

where $L^{\mu}$ are the $N$-dimensional irreducible representation matrices of the $\mathrm{SU}(2)$ generators. The normalization factor is just chosen so that the sphere has the unit radius, namely, $\left(X^{\mu}\right)^{2}=1$. The matrices satisfy the commutation relation,

$$
\left[X^{\mu}, X^{\nu}\right]=\epsilon^{\mu \nu \rho} \frac{2 i}{\sqrt{N^{2}-1}} X_{\rho}=\epsilon^{\mu \nu \rho} \frac{2 i}{N} X_{\rho}+\mathcal{O}\left(1 / N^{3}\right) .
$$

Hence the condition (1.1) is satisfied, where $C_{N}=N$.

The ground state energy of the Hamiltonian is given by

$$
E_{0}(y)=\frac{1}{2}\left(1+|y|^{2}\right)-\frac{N}{\sqrt{N^{2}-1}}|y|
$$

where $|y|=\sqrt{y_{\mu} y^{\mu}}$. The function $(2.3)$ is given by $f(y)=\frac{1}{2}(1-|y|)^{2}$, so that the classical space (2.2) is $S^{2}$ with the unit radius. The ground states are given by the so-called Bloch coherent states. See [11] for a detailed description.

By using the definition of the coherent states and the expressions such as (2.8), we can easily derive explicit forms of the geometric objects. They are given as follows.

$$
\begin{aligned}
P_{\nu}^{\mu}(y) & =\frac{1}{|y|}\left(\delta_{\nu}^{\mu}-\frac{y^{\mu} y_{\nu}}{|y|^{2}}\right), \\
W^{\mu \nu}(y) & =2 \epsilon^{\mu \nu \rho} \frac{y_{\rho}}{|y|}, \\
\omega_{\mu \nu}(y) & =-\frac{1}{2} \epsilon_{\mu \nu \rho} \frac{y^{\rho}}{|y|^{3}}, \\
J^{\mu}{ }_{\nu}(y) & =\epsilon^{\mu \nu \rho} \frac{y_{\rho}}{|y|^{2}}, \\
\tilde{g}_{\mu \nu}(y) & =\frac{1}{2|y|^{2}}\left(\delta_{\mu \nu}-\frac{y_{\mu} y_{\nu}}{|y|^{2}}\right) .
\end{aligned}
$$

We can see that for $y \in \mathcal{M}$ (i.e. for $|y|=1$ ), they satisfy the required properties such as (3.8), (3.13) and so on.

\section{Relation to the geometric quantization}

In this section, we show that when $C_{N}$ in (1.1) satisfies a certain condition, the matrix sequence we have considered can be semi-classically equivalent to a sequence obtained through the geometric quantization of the Kähler manifold. In appendix E, we review the geometric quantization scheme for a compact Kähler manifold. The main objects in the geometric quantization are the holomorphic sections of a complex line bundle, which corresponds to wave functions of the quantum mechanics, and physical observables represented as some differential operators on the wave functions. 
We first show that in the large- $N$ limit the ground states of the Hamiltonian behave as holomorphic sections, where the connection of the line bundle is given by the Berry connection. Let us consider an arbitrary antiholomorphic vector field $B^{\mu}$ satisfying

$$
J^{\mu}{ }_{\nu}(y) B^{\nu}(y)=-i B^{\mu}(y) . \quad(y \in \mathcal{M})
$$

The covariant derivative of $\langle\psi \mid 0, y\rangle$ along this vector field, where $\langle\psi|$ is any normalized state independent of $y$, is vanishing in the large- $N$ limit as we will see below. Firstly, from the definition of the covariant derivative, we have

$$
D_{\mu}\langle\psi \mid 0, y\rangle=\sum_{n \neq 0} \frac{\langle\psi \mid n, y\rangle\left\langle n, y\left|X_{\mu}\right| 0, y\right\rangle}{E_{n}(y)-E_{0}(y)} .
$$

Here recall that as one can see from (B.1), multiplying the Poisson tensor on $\left\langle n, y\left|X^{\mu}\right| 0, y\right\rangle$ gives a factor of $-i C_{N}\left(E_{n}(y)-E_{0}(y)\right)$ for $y \in \mathcal{M}$ in the large- $N$ limit. Thus, we obtain

$$
J^{\mu}{ }_{\nu}(y) D_{\mu}\langle\psi \mid 0, y\rangle \sim i D_{\nu}\langle\psi \mid 0, y\rangle,
$$

where $\sim$ stands for an equality for leading-order terms in the large- $N$ limit. By using this relation, we find that

$$
B^{\mu} D_{\mu}\langle\psi \mid 0, y\rangle=i B^{\nu} J_{\nu}^{\mu} D_{\mu}\langle\psi \mid 0, y\rangle \sim-B^{\nu} D_{\nu}\langle\psi \mid 0, y\rangle \sim 0 .
$$

Thus in the large- $N$ limit, $\langle\psi \mid 0, y\rangle$ behaves as a holomorphic section.

Secondly, we show that matrix elements of $X^{\mu}$ can be represented as the form of operators in the geometric quantization, which act on the holomorphic sections. We use the following relation:

$$
\begin{aligned}
\frac{i}{C_{N}} W^{\mu \nu}(y) D_{\nu}\langle\psi \mid 0, y\rangle & \sim \sum_{n \neq 0}\langle\psi \mid n, y\rangle\left\langle n, y\left|X^{\mu}\right| 0, y\right\rangle \\
& \sim\left\langle\psi\left|X^{\mu}\right| 0, y\right\rangle-\langle\psi \mid 0, y\rangle\left\langle 0, y\left|X^{\mu}\right| 0, y\right\rangle .
\end{aligned}
$$

This relation (5.5) can be rewritten into

$$
\left\langle\psi\left|X^{\mu}\right| 0, y\right\rangle \sim\left(y^{\mu}+\frac{i}{C_{N}} W^{\mu \nu}(y) D_{\mu}\right)\langle\psi \mid 0, y\rangle
$$

where we have used (2.9). This form is equal to the prequantum operator for the embedding function $y: \mathcal{M} \rightarrow R^{D}$ obtained by the geometric quantization. Furthermore, by multiplying $B^{\mu} D_{\mu}$ to the above equation, where $B^{\mu}$ is any antiholomorphic vector field, we see that the left-hand side is vanishing as shown in (5.4). Thus, we find that the righthand side is also vanishing under the action of $B^{\mu} D_{\mu}$ at least in the large- $N$ limit. This shows that the action of the prequantum operator is closed in the space of the holomorphic sections of the form $\langle\psi \mid 0, y\rangle$ in the large- $N$ limit.

These structures are very similar to the construction in the geometric quantization. However, we should note the following point. In our setup, the monopole charge of the Berry connection is of order of $C_{N}$ as we discussed in section 3.2. Let us write $C_{N}=c N^{a}$ so 
that the constant $a$ represents the order of the monopole charge. As shown in appendix E, the dimension of the Hilbert space spanned by all square integrable holomorphic sections is then of $\mathcal{O}\left(N^{a\left(\operatorname{dim}_{C} \mathcal{M}\right)}\right)$ in the large- $N$ limit, where $\operatorname{dim}_{C} \mathcal{M}$ is the complex dimension of $\mathcal{M}$. On the other hand, the dimension of the space spanned by the holomorphic sections of the form $\langle\psi \mid 0, y\rangle$ is at most $N$. Thus, we generally have

$$
1 / \operatorname{dim}_{C} \mathcal{M} \leq a
$$

The equality can be satisfied if any holomorphic section can be written in the form $\langle\psi \mid 0, y\rangle$ and the ground states $\{|0, y\rangle \mid y \in \mathcal{M}\}$ span the entire $N$-dimensional vector space.

If the equality holds in (5.7), the matrix elements (5.6) can be a true operator over the entire Hilbert space of the holomorphic sections. In this case, $X^{\mu}$ are semi-classically equivalent to the operators obtained from the geometric quantization. This case includes well-known examples such as the fuzzy torus and fuzzy sphere. On the other hand, if the equality is not satisfied, the ground states of the Hamiltonian are not sufficient to span the whole quantum Hilbert space in the geometric quantization.

\section{$6 \quad$ Summary and discussions}

In this paper, we considered the classical (commutative) limit of matrix geometry described by a sequence of $D$ Hermitian matrices, $\left\{\left(X_{1}^{(N)}, \cdots, X_{D}^{(N)}\right) \mid N \in I\right\}$. We adopted the formulation in [11] to describe the classical space and its tangent space. The classical space $\mathcal{M}$ is defined in terms of the Hamiltonian (2.1) and is given by the loci of zeros of the ground state energy in the large- $N$ limit as (2.2), while the tangent space of $\mathcal{M}$ is characterized by the projection matrix (2.5). We focused on the case where $\mathcal{M}$ is a smooth manifold and the matrices satisfy (1.1). We showed that in this case the classical space possesses a Kähler structure. Under the assumption that the ground state energy is nondegenerate on $\mathcal{M}$, we found formulas (2.8), (3.7), (3.18) and (3.20), which explicitly relate geometric structures and the matrix configurations. We also argued that when (1.1) is satisfied and the constant $C_{N}$ satisfies the equality in (5.7), the matrix configurations can be semi-classically equivalent to those obtained from the geometric quantization.

The inequality (5.7) shows a lower bound of the order of $C_{N}$ in the large- $N$ limit. We expect that there must also be an upper bound beyond which one of our assumptions is no longer satisfied. For example, consider the case where $a$ in (5.7) is infinity, this corresponds to situations where the matrices $X^{\mu}$ are commuting with each other even at finite $N$. In this case, the classical space is just a discrete set of points made of $N$ eigenvalues of the matrices. Thus, we do not have the notion of the tangent space etc. The upper bound of $a$ within which the classical space forms a smooth manifold should also be clarified, though this is beyond the scope of this paper.

In this paper, we have considered only the case where the ground state of the Hamiltonian is nondegenerate on the classical space $\mathcal{M}$. Though we think this should be a technical assumption and able to be removed without any considerable change of the method, there is one thing which we should take into account in the degenerate case. If ground states are degenerate, the Berry phase is allowed to take values in the Lie algebra of the special 
unitary group with a higher rank. In this case, we need to deal with the non-abelian gauge fields, as is expected from the viewpoint of D-branes in string theory. It would be interesting to investigate the matrix geometry in this case to gain a deeper understanding of multiple D-brane system in string theory.

The geometric objects defined in this paper provide a class of observables in matrix models, since they are invariant under the gauge transformations $X^{\mu} \rightarrow U X^{\mu} U^{\dagger}$. These observables capture geometric information of matrix models. For example, it is an interesting problem to consider a perturbation around some fixed matrix configuration and see how the geometric structures in matrix models are affected by the perturbation.

The perturbative calculation of the geometric structures is able to be carried out in our setting in the large- $N$ limit. In order to calculate them without any perturbation or extrapolation of the matrix size, however, generalization of our work to the case with a finite fixed matrix size seems to be needed (Recently, this has been partially done in [8].). This would make it possible to directly compute the geometric objects in matrix models by performing Monte Carlo simulations for example [28, 29].

There are also various interesting attempts to understand how the matrix models describe gravity [30-34]. It is important to consider whether and how our results can be applied in these contexts.

We hope to report on these issues in the near future.

\section{Acknowledgments}

This work was supported, in part, by Program to Disseminate Tenure Tracking System, MEXT, Japan.

\section{A Projectivity of $P^{\mu}{ }_{\nu}$}

In this appendix, we show that $P^{\mu}{ }_{\nu}$ defined in $(2.5)$ is a projection from vectors on $R^{D}$ to tangent vectors on $\mathcal{M}$.

Firstly, we write $P_{\nu}^{\mu}(y)$ at $y \in \mathcal{M}$ as

$$
P_{\nu}^{\mu}(y)=\delta_{\nu}^{\mu}-\lim _{N \rightarrow \infty} \partial_{\nu}\left\langle 0, y\left|y^{\mu}-X^{\mu}\right| 0, y\right\rangle .
$$

Since $\left\langle 0, y\left|y^{\mu}-X^{\mu}\right| 0, y\right\rangle$ is vanishing for any $y \in \mathcal{M}$ in the large- $N$ limit (see (2.9)), its derivatives along tangent directions are also vanishing. Thus, for a tangent vector $\epsilon_{\|}^{\mu}$ at $y \in \mathcal{M}$, we obtain

$$
P^{\mu}{ }_{\nu}(y) \epsilon_{\|}^{\nu}=\epsilon_{\|}^{\mu} .
$$

This shows that $P^{\mu}{ }_{\nu}$ is closed on tangent vectors and is equal to $\delta_{\nu}^{\mu}$.

Then, let $\epsilon_{\perp}^{\mu}$ be a normal vector at $y \in \mathcal{M}$. Below, we will show that

$$
P^{\mu}{ }_{\nu}(y) \epsilon_{\perp \mu} \epsilon_{\perp}^{\nu}=0 .
$$

From (A.2) and (A.3), the projectivity of $P^{\mu}{ }_{\nu}(y)$ follows immediately. Note that the lefthand side of (A.3) is obtained by computing $f\left(y+\epsilon_{\perp}\right)$. Namely, when $f\left(y+\epsilon_{\perp}\right)$ is expanded 
in a power series in $\epsilon_{\perp}$, the leading term is equal to $\frac{1}{2}\left(\delta_{\nu}^{\mu}-P_{\nu}^{\mu}(y)\right) \epsilon_{\perp \mu} \epsilon_{\perp}^{\nu}$, which contains the left-hand side of (A.3). On the other hand, $f\left(y+\epsilon_{\perp}\right)$ can also be evaluated as

$$
f\left(y+\epsilon_{\perp}\right)=\frac{1}{2}\left|\epsilon_{\perp}\right|^{2}+\mathcal{O}\left(\left|\epsilon_{\perp}\right|^{3}\right) .
$$

This leads to (A.3). In the following, we prove (A.4)

In order to prove (A.4), we first prove

$$
\lim _{N \rightarrow \infty} K_{N}\left\langle 0, y_{1}|H(y)| 0, y_{2}\right\rangle=\frac{1}{2}\left(y_{2}^{\mu}-y^{\mu}\right)^{2} \delta^{(D)}\left(y_{1}-y_{2}\right), \quad\left(y_{1}, y_{2} \in \mathcal{M}\right)
$$

where $K_{N}$ is a positive constant defined below. The object on the left-hand side can be computed as

$$
\left\langle 0, y_{1}|H(y)| 0, y_{2}\right\rangle=\left(E_{0}\left(y_{2}\right)+\frac{1}{2}\left(y_{2}^{\mu}-y^{\mu}\right)^{2}\right)\left\langle 0, y_{1} \mid 0, y_{2}\right\rangle+\left(y_{2}^{\mu}-y^{\mu}\right)\left\langle 0, y_{1}\left|X_{\mu}-y_{2 \mu}\right| 0, y_{2}\right\rangle .
$$

Because of (2.9), the inner product $\left\langle 0, y_{1} \mid 0, y_{2}\right\rangle$ satisfies

$$
\lim _{N \rightarrow \infty}\left(y_{2}^{\mu}-y_{1}^{\mu}\right)\left\langle 0, y_{1} \mid 0, y_{2}\right\rangle=0 . \quad\left(y_{1}, y_{2} \in \mathcal{M}\right)
$$

This implies that the ground states at different points are orthogonal to each other in the large- $N$ limit. So we obtain

$$
\lim _{N \rightarrow \infty} K_{N}\left\langle 0, y_{1} \mid 0, y_{2}\right\rangle=\delta^{(D)}\left(y_{1}-y_{2}\right) . \quad\left(y_{1}, y_{2} \in \mathcal{M}\right)
$$

Here, we have introduced a proportionality constant $K_{N}$. By applying (2.9) and (A.8) to (A.6), we obtain (A.5).

Then, we prove (A.4). Note that $f\left(y+\epsilon_{\perp}\right)$ can be written as

$$
f\left(y+\epsilon_{\perp}\right)=\lim _{N \rightarrow \infty} \min _{|\alpha\rangle \in \mathcal{H}}\left\langle\alpha\left|H\left(y+\epsilon_{\perp}\right)\right| \alpha\right\rangle,
$$

where $|\alpha\rangle$ shall be normalized as $\langle\alpha \mid \alpha\rangle=1$. Let us denote by $\mathcal{H}_{\mathrm{c}}$ the Hilbert space spanned by all the ground states in $\mathcal{M}$ :

$$
\mathcal{H}_{\mathrm{c}}=\operatorname{span}\left\{\left|0, y^{\prime}\right\rangle \mid y^{\prime} \in \mathcal{M}\right\} \text {. }
$$

The total space can then be decomposed as

$$
\mathcal{H}=\mathcal{H}_{\mathrm{c}} \oplus \tilde{\mathcal{H}} .
$$

By definition, $\tilde{\mathcal{H}}$ is the subspace on which the eigenvalues of Hamiltonian are always nonvanishing. Any element $|\beta\rangle$ in $\mathcal{H}_{\mathrm{c}}$ can be expanded as

$$
|\beta\rangle=\sqrt{K_{N}} \int d^{D} y^{\prime} \beta\left(y^{\prime}\right)\left|0, y^{\prime}\right\rangle,
$$

where $\beta\left(y^{\prime}\right)$ is a function which is vanishing unless $y^{\prime} \in \mathcal{M}$. Then a generic normalized vector $|\alpha\rangle$ takes the form,

$$
|\alpha\rangle=|\beta\rangle+|\tilde{\alpha}\rangle,
$$


where $|\beta\rangle$ is given by (A.12) and $|\tilde{\alpha}\rangle \in \tilde{\mathcal{H}}$. The normalization condition is

$$
\langle\beta \mid \beta\rangle+\langle\tilde{\alpha} \mid \tilde{\alpha}\rangle=1 .
$$

By substituting (A.13) into (A.9), we obtain

$$
\begin{aligned}
f\left(y+\epsilon_{\perp}\right)=\lim _{N \rightarrow \infty} \min _{|\beta\rangle,|\tilde{\alpha}\rangle}\left[\left\langle\beta\left|H\left(y+\epsilon_{\perp}\right)\right| \beta\right\rangle+\left\langle\tilde{\alpha}\left|H\left(y+\epsilon_{\perp}\right)\right| \beta\right\rangle\right. \\
\left.+\left\langle\beta\left|H\left(y+\epsilon_{\perp}\right)\right| \tilde{\alpha}\right\rangle+\left\langle\tilde{\alpha}\left|H\left(y+\epsilon_{\perp}\right)\right| \tilde{\alpha}\right\rangle\right],
\end{aligned}
$$

For any $|\beta\rangle$ and $|\tilde{\alpha}\rangle$, the second and the third terms are vanishing in the large- $N$ limit because of (2.10) and the orthogonality between $|\beta\rangle$ and $|\tilde{\alpha}\rangle$. Here, let us consider the case where $\left|\epsilon_{\perp}\right|$ is very small and put

$$
\langle\beta \mid \beta\rangle=c\left|\epsilon_{\perp}\right|^{a},
$$

where $a$ and $c$ are non-negative constants. Then, the last term in (A.15) is

$$
\left(1-c\left|\epsilon_{\perp}\right|^{a}\right) \frac{\left\langle\tilde{\alpha}\left|H\left(y+\epsilon_{\perp}\right)\right| \tilde{\alpha}\right\rangle}{\langle\tilde{\alpha} \mid \tilde{\alpha}\rangle} .
$$

Note that from the definition of $\tilde{\mathcal{H}}$, the second factor is positive definite and is $\mathcal{O}\left(\left|\epsilon_{\perp}\right|^{0}\right)$. Hence, (A.17) is $\mathcal{O}\left(\left|\epsilon_{\perp}\right|^{0}\right)$ unless $a=0$ and $c=1$. Similarly, by using (A.5), the first term in (A.15) can be estimated as $\mathcal{O}\left(\left|\epsilon_{\perp}\right|^{a+2}\right)$. Thus, we find that the minimum in (A.15) is saturated when $a=0$ and $c=1$. (A.15) is now reduced to

$$
f\left(y+\epsilon_{\perp}\right)=\lim _{N \rightarrow \infty} \min _{|\beta\rangle \in \mathcal{H}_{\mathrm{c}}}\left\langle\beta\left|H\left(y+\epsilon_{\perp}\right)\right| \beta\right\rangle .
$$

Finally, by substituting the expansion (A.12) into (A.18) and using (A.5), we obtain

$$
f\left(y+\epsilon_{\perp}\right)=\frac{1}{2} \int d^{D} y^{\prime}\left|\hat{\beta}\left(y^{\prime}\right)\right|^{2}\left(y^{\prime \mu}-y^{\mu}-\epsilon_{\perp}^{\mu}\right)^{2} .
$$

Here, $\hat{\beta}\left(y^{\prime}\right)$ is the large- $N$ limit of the function which saturates the minimum in (A.18). It satisfies $\int d^{D} y^{\prime}\left|\hat{\beta}\left(y^{\prime}\right)\right|^{2}=1$ and vanishes on the outside of $\mathcal{M}$. Obviously, the quantity on the right-hand side of (A.19) is minimized when $\hat{\beta}\left(y^{\prime}\right)$ localizes at $y$. Thus, we finally obtain (A.4).

\section{B Properties of $W^{\mu \nu}$}

\section{B.1 Proof of (3.2)}

Here, we show that $W^{\mu \nu}(y)$ is a tangent bivector, namely, it satisfies (3.2). To this end, we use the following relation:

$$
\left\langle 0, y\left|\left[X^{\mu}, X^{\nu}\right]\right| 0, y\right\rangle\left\langle 0, y\left|X_{\nu}\right| n, y\right\rangle \sim\left(E_{n}(y)-E_{0}(y)\right)\left\langle 0, y\left|X^{\mu}\right| n, y\right\rangle,
$$

where $\sim$ stands for an equality for the leading-order terms in the large- $N$ limit. The relation (B.1) is shown as follows. By using (1.1) and (2.9), we obtain

$$
\left\langle 0, y\left|\left[X^{\mu}, X^{\nu}\right]\right| 0, y\right\rangle\left\langle 0, y\left|X_{\nu}\right| n, y\right\rangle \sim \frac{1}{2}\left\langle 0, y\left|\left\{X^{\nu}-y^{\nu},\left[X^{\mu}, X_{\nu}-y_{\nu}\right]\right\}\right| n, y\right\rangle .
$$


Then, note that $\left\{X^{\nu}-y^{\nu},\left[X^{\mu}, X_{\nu}-y_{\nu}\right]\right\}=2\left[X^{\mu}, H(y)\right]$. By substituting this into (B.2) we obtain (B.1). Now, let us calculate

$$
P_{\rho}^{\mu}(y) W^{\rho \nu}(y)=\lim _{N \rightarrow \infty}\left[2 C_{N} \operatorname{Im}\left\{\sum_{n \neq 0} \frac{\left\langle 0, y\left|X^{\mu}\right| n, y\right\rangle\left\langle n, y\left|X^{\rho}\right| 0, y\right\rangle}{E_{n}(y)-E_{0}(y)}\left\langle 0, y\left|\left[X_{\rho}, X^{\nu}\right]\right| 0, y\right\rangle\right\}\right] .
$$

By using (B.1) and the completeness relation $\sum_{n=0}^{N-1}|n, y\rangle\langle n, y|=1$, we obtain

$$
P_{\rho}^{\mu}(y) W^{\rho \nu}(y)=\lim _{N \rightarrow \infty}\left[2 C_{N} \operatorname{Im}\left\{\left\langle 0, y\left|X^{\mu} X^{\nu}\right| 0, y\right\rangle-\left\langle 0, y\left|X^{\mu}\right| 0, y\right\rangle\left\langle 0, y\left|X^{\nu}\right| 0, y\right\rangle\right\}\right] .
$$

The second term is zero since $X^{\mu}$ are Hermitian, while the first term is equal to $W^{\mu \nu}(y)$. Thus, we have shown (3.2).

\section{B.2 Proof of Jacobi identity}

Here, we show that the bracket defined in (3.3) satisfies the Jacobi identity. The Jacobi identity is equivalent to

$$
W^{\mu \nu}(y) \partial_{\mu} W^{\rho \sigma}(y)+W^{\mu \rho}(y) \partial_{\mu} W^{\sigma \nu}(y)+W^{\mu \sigma}(y) \partial_{\mu} W^{\nu \rho}(y)=0,
$$

for $y \in \mathcal{M}$.

Let us consider an arbitrary polynomial $\Phi(X)$, where the coefficients and degree are assumed to be $N$-independent, and let us define a corresponding function by

$$
\Phi(y)=\lim _{N \rightarrow \infty}\langle 0, y|\Phi(X)| 0, y\rangle
$$

Then, as shown below, the following relation holds:

$$
\lim _{N \rightarrow \infty}\left(-i C_{N}\right)\left\langle 0, y\left|\left[X^{\mu}, \Phi(X)\right]\right| 0, y\right\rangle=W^{\mu \nu}(y) \partial_{\nu} \Phi(y)
$$

If we put $\Phi(X)=W^{\mu \nu}(X)=-i C_{N}\left[X^{\mu}, X^{\nu}\right]+\mathcal{O}\left(1 / C_{N}\right)$ in the above equation, (B.5) immediately follows from the Jacobi identity of the matrix commutators.

Below, we show the relation (B.7). The right-hand side of (B.7) is the large- $N$ limit of

$$
-i C_{N}\left\langle 0, y\left|\left[X^{\mu}, X^{\nu}\right]\right| 0, y\right\rangle \partial_{\nu}\langle 0, y|\Phi(X)| 0, y\rangle
$$

The derivative of $|0, y\rangle$ is obtained from the formula in the perturbation theory as

$$
\partial_{\mu}|0, y\rangle=\sum_{n \neq 0} \frac{|n, y\rangle\left\langle n, y\left|X_{\mu}\right| 0, y\right\rangle}{E_{n}(y)-E_{0}(y)}+i A_{\mu}(y)|0, y\rangle
$$

where $A_{\mu}(y)$ is the Berry connection. By substituting this, and using (B.1), we find that the large- $N$ limit of (B.8) is also equal to the left-hand side of (B.7). Thus, we obtain (B.7). 


\section{Construction of compatible structures}

In this appendix, we show that on a symplectic manifold, if an arbitrary metric is given, one can find an almost complex structure and a Riemann structure compatible with the symplectic form.

We assume that we are given a metric $g$ on a symplectic manifold $(\mathcal{M}, \omega)$. For any tangent vector $u \in T \mathcal{M}$, let us consider two mappings defined by

$$
\begin{aligned}
& u \mapsto \omega(u, \cdot) \in T^{*} \mathcal{M}, \\
& u \mapsto g(u, \cdot) \in T^{*} \mathcal{M} .
\end{aligned}
$$

Since both of $g$ and $\omega$ are nondegenerate, these mappings define isomorphisms $T \mathcal{M} \rightarrow$ $T^{*} \mathcal{M}$. Then we can define an isomorphism $A: T \mathcal{M} \rightarrow T \mathcal{M}$ by

$$
\omega(u, v)=g(A u, v) . \quad(u, v \in T \mathcal{M})
$$

The isomorphism $A$ is antisymmetric, since

$$
g\left(A^{T} u, v\right)=g(u, A v)=g(A v, u)=\omega(v, u)=-\omega(u, v)=-g(A u, v) .
$$

The combination $A A^{T}$ is symmetric. By diagonalizing it, we can write

$$
A A^{T}=B \operatorname{diag}\left(\lambda_{1}, \lambda_{2}, \cdots, \lambda_{\operatorname{dim} \mathcal{M}}\right) B^{-1},
$$

where $\lambda_{i}>0(i=1,2, \cdots, \operatorname{dim} \mathcal{M})$.

An almost complex structure $J$ on $\mathcal{M}$ can be defined by a polar decomposition of $A$ as

$$
J=\left(A A^{T}\right)^{-1 / 2} A,
$$

where $\left(A A^{T}\right)^{a}$ is defined by replacing $\lambda_{i}$ with $\lambda_{i}^{a}$ in the right-hand side of (C.4). Obviously, $J$ is antisymmetric and satisfies $J^{2}=-1$.

In general, an almost complex structure is said to be compatible with the symplectic structure if for any $u, v \in T \mathcal{M}$ it satisfies

$$
\omega(J u, J v)=\omega(u, v)
$$

and

$$
\omega(u, J u)>0 .
$$

It is easy to see that the definition (C.5) satisfies the both conditions and thus it gives a compatible almost complex structure. In fact, one can check the first condition by

$$
\omega(J u, J v)=g(A J u, J v)=g(J A u, J v)=g\left(A u, J^{T} J v\right)=g(A u, v)=\omega(u, v),
$$

and the second condition by

$$
\omega(u, J u)=g(A u, J u)=-g(J A u, u)=g\left(\left(A A^{T}\right)^{1 / 2} u, u\right)=g\left(\left(A A^{T}\right)^{1 / 4} u,\left(A A^{T}\right)^{1 / 4} u\right)>0 .
$$

Once a compatible pair of the symplectic form and almost complex structure $(\omega, J)$ is given, one can define a new compatible metric $\tilde{g}$ by

$$
\tilde{g}(u, v)=\omega(u, J v) .
$$

The positivity is guaranteed by (C.7). 


\section{Explicit forms of $J$ and $\tilde{g}$}

In this appendix, we derive (3.18) and (3.20).

Because of (3.2), the complex structure $J$ and the metric $\tilde{g}$ can be written for $y \in \mathcal{M}$ as

$$
\begin{aligned}
& J(y)=\frac{1}{\sqrt{W(y) W^{T}(y)}} W(y) P(y), \\
& \tilde{g}(y)=\frac{1}{\sqrt{W(y) W^{T}(y)}} P(y) .
\end{aligned}
$$

Here, let us consider the equation (B.1). This shows that the multiplication of $W$ on $\left\langle 0, y\left|X^{\nu}\right| n, y\right\rangle$ produces the factor $-i C_{N}\left(E_{n}(y)-E_{0}(y)\right)$. This also implies that the multiplication of $\frac{1}{\sqrt{W W^{T}}}$ yields $\frac{1}{C_{N}\left(E_{n}(y)-E_{0}(y)\right)}$. Then, applying these calculation to (D.1), where $P$ is written as (2.8), we obtain (3.18) and (3.20).

Note that (B.1) implies that $E_{n}-E_{0}=\mathcal{O}\left(1 / C_{N}\right)$ or otherwise $\left\langle 0, y\left|X^{\mu}\right| n, y\right\rangle=0$. So the above multiplication is well-defined in the large- $N$ limit.

\section{E Geometric quantization on Kähler manifold}

In this appendix, we briefly review the geometric quantization on a compact Kähler manifold $[5,6]$. The geometric quantization is a generalization of the process of up-lifting a classical mechanics to the corresponding quantum mechanics. In the usual setup of Hamilton mechanics, the classical phase space is an even dimensional flat space. The coordinates $\left(p_{i}, q_{i}\right)$ and observables $f_{n}(p, q)$ are promoted to some Hermitian operators in going from the classical mechanics to the quantum mechanics. The geometric quantization is a generalization of this problem such that the phase space at the starting point is not a flat space but a non-trivial symplectic manifold. The goal of the geometric quantization is to construct a quantum Hilbert space and quantum mechanical operators represented on that space, for given symplectic manifold $\mathcal{M}$ and classical observables on $\mathcal{M}$.

We consider a Kähler manifold, which has a compatible triple $(\omega, J, \tilde{g})$, where $\omega, J$ and $\tilde{g}$ are symplectic, complex and Riemann structures, respectively. For simplicity, let $\omega$ be normalized in such a way that $\omega / 2 \pi$ represents an integral cohomology class and its integral over every closed two-cycle in $\mathcal{M}$ is equal to 1 . At least locally (i.e. on each coordinate patch), one can introduce the symplectic potential as

$$
\omega=d \theta \text {. }
$$

On an overlap of two patches, two coordinates are related by a symplectomorphism preserving $\omega$. Under the symplectomorphism, $\theta$ transforms like a $\mathrm{U}(1)$ gauge field. If $\mathcal{M}$ possesses non-trivial one-cycles, there will be an ambiguity in the definition of $\theta$ coming from the choice of the holonomy. For each choice, the following construction works. So we take one of them and fix it in the following discussion.

We first introduce the notion of the prequantum line bundle. In the case of quantum mechanics (i.e. when $\mathcal{M}$ is flat,) wave functions usually depend only on $q_{i}$, or in the momentum representation, only on $p_{i}$. The sections of the prequantum line bundle correspond to more general functions of the form $\psi\left(p_{i}, q_{i}\right)$ and form a larger reducible Hilbert space. 
In order to obtain a relevant Hilbert space, one needs a truncation to the irreducible space. This procedure (choosing a polarization) is discussed later. To define the prequantum line bundle, let us introduce covariant derivatives as

$$
D_{a}=\partial_{a}-i A_{a}
$$

Here the new gauge field $A$ is defined by

$$
A=n \theta,
$$

where $n$ is an arbitrary fixed integer. We denote by $F$ the curvature of $A$. The integer $n$ then corresponds to the monopole charge of $F$. The prequantum line bundle is just the associated complex line bundle with the curvature given by $F$. Let $\Phi$ be a section of the line bundle. It transforms as $\Phi \rightarrow e^{i \Lambda} \Phi$ when the gauge field transforms as $A \rightarrow A+d \Lambda$. The inner products for sections are defined by using the Liouville measure of $\omega$.

Next, we introduce prequantum operators. For a given function $f$ on the Kähler manifold, the prequantum operator is defined by

$$
\mathcal{P}(f)=f-i \xi_{f}^{a} D_{a},
$$

where $\xi_{f}^{a}$ is the Hamilton vector field of $f$ defined by

$$
i_{\xi_{f}} \omega=-d f
$$

The prequantum operators act on the space of sections introduced above. One of the most important properties of those operators is

$$
[\mathcal{P}(f), \mathcal{P}(g)]=i \mathcal{P}(\{f, g\}),
$$

where, $\{$,$\} is the Poisson bracket defined by$

$$
\{f, g\}=i_{\xi_{f}} i_{\xi_{g}} \omega
$$

Namely, the Poisson bracket is mapped to the commutator under the action of $\mathcal{P}$.

In order to obtain a relevant irreducible Hilbert space, one needs to choose a polarization of the wave functions. On Kähler manifolds, one can take the so-called holomorphic polarization. By using the complex structure, we can define holomorphic and anti-holomorphic covariant derivatives,

$$
D_{z_{i}}=\partial_{z_{i}}-i A_{z_{i}}, \quad D_{\bar{z}_{i}}=\partial_{\bar{z}_{i}}-i A_{\bar{z}_{i}},
$$

where $i$ runs from 1 to the complex dimension of the manifold. The polarization condition is then written as

$$
D_{\bar{z}_{i}} \Phi=0,
$$

for any $i$, where $\Phi$ is a section of the prequantum line bundle. Square integrable holomorphic sections satisfying (E.9) form a subspace of the prequantum Hilbert space and this defines the true quantum Hilbert space. The quantum Hilbert space generally forms an irreducible representation space of the Poisson algebra. 
The dimension of the quantum Hilbert space is finite in general for the Kähler polarization on a compact Kähler manifold. Through an index theorem, the dimension is given by an integral over $\mathcal{M}$ of a wedge product of the Todd class on $\mathcal{M}$ and the Chern character of $F$. For example, a Riemann surface with genus $g$, the dimension is given by $n+1-g$.

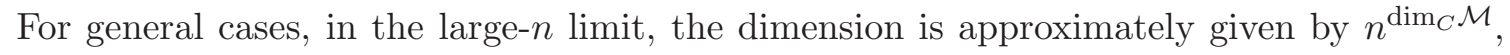
where $\operatorname{dim}_{C} \mathcal{M}$ is the complex dimension of $\mathcal{M}$.

If a prequantum operator is closed on the polarized wave functions, it can be promoted to an operator on the polarized Hilbert space. If this is not the case, however, things become a little complicated. For such cases, prescriptions to construct operators in the polarized space are known for some particular cases (see $[5,6]$ and references therein.), though we will not treat those cases in this paper.

Open Access. This article is distributed under the terms of the Creative Commons Attribution License (CC-BY 4.0), which permits any use, distribution and reproduction in any medium, provided the original author(s) and source are credited.

\section{References}

[1] T. Banks, W. Fischler, S.H. Shenker and L. Susskind, M theory as a matrix model: a conjecture, Phys. Rev. D 55 (1997) 5112 [hep-th/9610043] [INSPIRE].

[2] N. Ishibashi, H. Kawai, Y. Kitazawa and A. Tsuchiya, A large- $N$ reduced model as superstring, Nucl. Phys. B 498 (1997) 467 [hep-th/9612115] [InSPIRE].

[3] B. de Wit, J. Hoppe and H. Nicolai, On the quantum mechanics of supermembranes, Nucl. Phys. B 305 (1988) 545 [InSPIRE].

[4] J. Madore, The fuzzy sphere, Class. Quant. Grav. 9 (1992) 69 [InSPIRE].

[5] N.M.J. Woodhouse, Geometric quantization, Clarendon Press, Oxford U.K. (1992).

[6] E. Lerman, Geometric quantization; a crash course, arXiv:1206.2334.

[7] D. Berenstein and E. Dzienkowski, Matrix embeddings on flat $R^{3}$ and the geometry of membranes, Phys. Rev. D 86 (2012) 086001 [arXiv:1204.2788] [INSPIRE].

[8] L. Schneiderbauer and H.C. Steinacker, Measuring finite quantum geometries via quasi-coherent states, J. Phys. A 49 (2016) 285301 [arXiv:1601.08007] [InSPIRE].

[9] T. Asakawa, S. Sugimoto and S. Terashima, D-branes, matrix theory and K homology, JHEP 03 (2002) 034 [hep-th/0108085] [INSPIRE].

[10] S. Terashima, Noncommutativity and tachyon condensation, JHEP 10 (2005) 043 [hep-th/0505184] [INSPIRE].

[11] G. Ishiki, Matrix geometry and coherent states, Phys. Rev. D 92 (2015) 046009 [arXiv: 1503.01230] [INSPIRE].

[12] J. Arnlind, J. Hoppe and G. Huisken, Multi-linear formulation of differential geometry and matrix regularizations, J. Diff. Geom. 91 (2012) 1 [arXiv:1009.4779] [InSPIRE].

[13] G. Alexanian, A.P. Balachandran, G. Immirzi and B. Ydri, Fuzzy $C P^{2}$, J. Geom. Phys. 42 (2002) 28 [hep-th/0103023] [InSPIRE].

[14] A. Connes, M.R. Douglas and A.S. Schwarz, Noncommutative geometry and matrix theory: compactification on tori, JHEP 02 (1998) 003 [hep-th/9711162] [INSPIRE]. 
[15] J. Arnlind, M. Bordemann, L. Hofer, J. Hoppe and H. Shimada, Fuzzy Riemann surfaces, JHEP 06 (2009) 047 [hep-th/0602290] [INSPIRE].

[16] J. Castelino, S. Lee and W. Taylor, Longitudinal five-branes as four spheres in matrix theory, Nucl. Phys. B 526 (1998) 334 [hep-th/9712105] [INSPIRE].

[17] H. Grosse and P. Prešnajder, The construction on noncommutative manifolds using coherent states, Lett. Math. Phys. 28 (1993) 239 [inSPIRE].

[18] H. Grosse and P. Prešnajder, The Dirac operator on the fuzzy sphere, Lett. Math. Phys. 33 (1995) 171 [INSPIRE].

[19] A.B. Hammou, M. Lagraa and M.M. Sheikh-Jabbari, Coherent state induced star product on $R_{\lambda}^{3}$ and the fuzzy sphere, Phys. Rev. D 66 (2002) 025025 [hep-th/0110291] [InSPIRE].

[20] A. Chatzistavrakidis, H. Steinacker and G. Zoupanos, Intersecting branes and a standard model realization in matrix models, JHEP 09 (2011) 115 [arXiv:1107.0265] [INSPIRE].

[21] M.H. de Badyn, J.L. Karczmarek, P. Sabella-Garnier and K. H.-C. Yeh, Emergent geometry of membranes, JHEP 11 (2015) 089 [arXiv: 1506.02035] [INSPIRE].

[22] J.L. Karczmarek and K. H.-C. Yeh, Noncommutative spaces and matrix embeddings on flat $R^{2 n+1}$, JHEP 11 (2015) 146 [arXiv:1506.07188] [INSPIRE].

[23] N. Seiberg and E. Witten, String theory and noncommutative geometry, JHEP 09 (1999) 032 [hep-th/9908142] [INSPIRE].

[24] R.C. Myers, Dielectric branes, JHEP 12 (1999) 022 [hep-th/9910053] [INSPIRE].

[25] G. Ishiki, S. Shimasaki, Y. Takayama and A. Tsuchiya, Embedding of theories with $\mathrm{SU}(2 \mid 4)$ symmetry into the plane wave matrix model, JHEP 11 (2006) 089 [hep-th/0610038] [INSPIRE].

[26] J.M. Maldacena, M.M. Sheikh-Jabbari and M. Van Raamsdonk, Transverse five-branes in matrix theory, JHEP 01 (2003) 038 [hep-th/0211139] [INSPIRE].

[27] T. Ishii, G. Ishiki, S. Shimasaki and A. Tsuchiya, $N=4$ super Yang-Mills from the plane wave matrix model, Phys. Rev. D 78 (2008) 106001 [arXiv:0807.2352] [INSPIRE].

[28] S. Catterall and T. Wiseman, Towards lattice simulation of the gauge theory duals to black holes and hot strings, JHEP 12 (2007) 104 [arXiv:0706.3518] [INSPIRE].

[29] K.N. Anagnostopoulos, M. Hanada, J. Nishimura and S. Takeuchi, Monte Carlo studies of supersymmetric matrix quantum mechanics with sixteen supercharges at finite temperature, Phys. Rev. Lett. 100 (2008) 021601 [arXiv:0707.4454] [INSPIRE].

[30] M. Hanada, H. Kawai and Y. Kimura, Describing curved spaces by matrices, Prog. Theor. Phys. 114 (2006) 1295 [hep-th/0508211] [INSPIRE].

[31] H. Steinacker, Emergent gravity from noncommutative gauge theory, JHEP 12 (2007) 049 [arXiv: 0708.2426] [INSPIRE].

[32] H. Steinacker, The curvature of branes, currents and gravity in matrix models, JHEP 01 (2013) 112 [arXiv: 1210.8364] [INSPIRE].

[33] Y. Ito, J. Nishimura and A. Tsuchiya, Power-law expansion of the universe from the bosonic Lorentzian type IIB matrix model, JHEP 11 (2015) 070 [arXiv:1506.04795] [INSPIRE].

[34] M. Hanada, Y. Hyakutake, G. Ishiki and J. Nishimura, Numerical tests of the gauge/gravity duality conjecture for D0-branes at finite temperature and finite $N$, arXiv: 1603.00538 [INSPIRE]. 